\title{
Association, direct and indirect effects of yield, biochemical and physiological characters on seed yield in chickpea (Cicer arietinum L.)
}

\section{Halavath Sai Kumar 夰}

Department of Genetics and Plant Breeding, Naini Agricultural institute, Sam Higginbottom University of Agriculture Technology \& Sciences, Prayagraj, Uttar Pradesh, India.

\section{Sindhuja Yerrabala}

Department of Agricultural Entomology, Naini Agricultural institute, Sam Higginbottom University of Agriculture Technology \& Sciences, Prayagraj, Uttar Pradesh, India

\section{G.R. Lavanya}

Department of Genetics and Plant Breeding, Naini Agricultural institute, Sam Higginbottom University of Agriculture Technology \& Sciences, Prayagraj, Uttar Pradesh, India

\section{G. Babithraj Goud}

Department of Crop Physiology, PJTSAU, Rajendranagar, Hyderabad, Telangana, India

\section{Y.Vinod Kumar Reddy}

Department of Genetics and Plant Breeding, Naini Agricultural institute, Sam Higginbottom University of Agriculture Technology \& Sciences, Prayagraj, Uttar Pradesh, India

\section{Varri Sharmila}

Department of Genetics and Plant Breeding, Naini Agricultural institute, Sam Higginbottom University of Agriculture Technology \& Sciences, Prayagraj, Uttar Pradesh, India

\begin{tabular}{|c|c|}
\hline ARTICLE INFO & ABSTRACT \\
\hline $\begin{array}{l}\text { Received : } 12 \text { May } 2021 \\
\text { Revised : } 22 \text { June } 2021 \\
\text { Accepted : } 30 \text { June } 2021 \\
\text { Available online: } 09 \text { December } 202 \\
\text { Key Words: } \\
\text { Chickpea } \\
\text { Correlation } \\
\text { Direct and Indirect effects } \\
\text { Genetic advance } \\
\text { Heritability } \\
\text { Genotypic variance } \\
\text { Phenotypic variance } \\
\text { Variability }\end{array}$ & $\begin{array}{l}\text { The present research consists of the } 25 \text { genotypes of chickpea (Cicer arietinum } \\
\text { L.) which was carried out at the field experimentation center, Department of } \\
\text { the Genetics and Plant Breeding, Naini Agricultural Institute, Sam } \\
\text { Higginbottom University of Agriculture, Technology and Sciences, Prayagraj } \\
\text { during Rabi } 2019-20 \text { in Randomized Block Design with three replications with } \\
\text { an aim to determine genetic variability, correlation, direct and indirect effects } \\
\text { yield, biochemical and physiological characters on seed yield. All the genotypes } \\
\text { of chickpea showed significant differences among them, significance variability } \\
\text { existed for all the characters. Based on the mean performance, high yield was } \\
\text { found for the C-18106 followed by C-18122, C-18103, and C-18101, genotypes } \\
\text { C-18123, ICC-15896, C-18102 \& GPF-02 showed against pod borer. High } \\
\text { heritability ( }>70 \% \text { ) coupled with high genetic advance ( }>20 \% \text { were being } \\
\text { observed for the number of seeds per plant, biological yield. Seed yield per } \\
\text { plant exhibited positive and highly significant correlation with biological yield, } \\
\text { chlorophyll index, starch content and number of seeds per plant at both } \\
\text { genotypic and phenotypic level. Path analysis at phenotypic level identified } \\
\text { hundred seed weight followed by chlorophyll index, number of seeds per plant, } \\
\text { number of secondary branches, trypsin inhibitor and harvest index important } \\
\text { direct components for seed yield per plant. Thus, due consideration should be } \\
\text { given to these characters during the selection. }\end{array}$ \\
\hline
\end{tabular}

\section{Introduction}

Legumes ecologically as well as economically important plants, are considered vital for global food security especially under predicted climatic conditions (Vavilov et al.,1926). Chickpea is a cool season legume crop and it grown in several countries worldwide as a food source (Fiaz et al.,
2016). Chickpea is the third most important food legume crop and India is the largest producer contributing to $65 \%$ of world's chickpea production (DOES, 2019): it imports chickpea from other countries. However the ever-increasing demand for this legume crop; it is essential to improve the

Corresponding author E-mail: saikumarhalavath@gmail.com

Doi: https://doi.org/10.36953/ECJ.2021.22321

This work is licensed under Attribution-Non Commercial 4.0 International (CC BY-NC 4.0)

(C) ASEA 
production and area under cultivation (Sain et al., 2020). The area under chickpea cultivation decreased due to lack of high yielding varieties and susceptibility to insect and diseases (Kumar et al., 2018). The yield of chickpea can be improved by selection of superior genotypes which is directly related with the seed yield and utilize these genotypes exclusively in breeding programs to enhance grain yield. Yield is the ultimate goal of a breeding program, (Sain et al., 2020; Muhammad et al., 2003). Seed yield being most important trait is governed by many physiological changes within the plant and influenced by many environmental factors so the breeder needs some index traits to select elite genotypes for higher yield (Manasa et al., 2019).

Seed yield is a polygenic and complex trait which is affected by a large number of other components, so direct selection based on association pattern alone between two variables many sometimes mislead the breeder hence it should split into direct and indirect effects for effective selection (Awol and Alise fikre, 2018; Dehal et al., 2016; Yadav et al.,1926). Genetic variation among traits is important for breeding and selecting desirable types on other hand an analysis of the correlation between seed yield and yield components is essential in determining selection criteria however path coefficient analysis helps to determine the direct effect of traits and their indirect effects on other traits (Arora and Jeena, 2001; Chopdar et al., 2017; Dewey and Lu,1959). Correlation does not provide the adequate picture of the relationship between the variables in addition to the degree of such relationship, path coefficient analysis measure the direct influence of one variable upon the other and permits separation of correlation coefficients into components of direct and indirect effects (Kaur and Bhardwaj, 2019; Maloo and Sharma,1987; Saroj et al.,2013). It is not sufficient to describe this relationship when the causal association among characteristics is needed. The plus point of this analysis that it allows the partitioning of correlation coefficient into its components (Dewey et al., 1959; Wright et al.,1921). Path coefficient analysis examines each and every component and provides information on cause of association between two traits if the association between yield and other characters is due to direct effect. It indicates true and perfect correlation between those two traits and selection would be effective for that character to improve seed yield (Singh et al., 2008; Kumar et al., 2019: Gediya et al., 2019).

\section{Material and Methods}

A genotypes collection of 25 strains of chickpea (Cicer arietinum L.) comprising indigenous as well as exotic genotypes, constituted the experimental materials for this study. These genotypes exhibiting wide spectrum of variability for various quantitative, biochemical and physiological characters were obtained from the pulse section, field experimentation center, Department of Genetics and Plant Breeding, Naini Agricultural Institute, Sam Higginbottom University of Agriculture Technology and Sciences, Prayagraj. The present experiment was carried out in Rabi 2019-20 in Randomized Block Design. The treatments were being replicated three times. The plot size of $1 * 1 \mathrm{~m}^{2}$ the row to row spacing $30 \mathrm{~cm}$ and plant to plant distance $10 \mathrm{~cm}$. Soil in this region is sandy loam and alkaline in nature. The technique of random sampling was adopted for the observation of the 25 quantitative, biochemical and physiological characters. Recommended practices were applied to raise a healthy crop. Metric data on 25 traits were taken at different stages of growth. The experimental data thus recorded on these characters were subjected to statistical and biometrical analysis for Analysis of variance (Fisher and Yates, 1963; Banik et al., 2018), different genetic parameters Coefficient of variation (GCV, PCV) (Burton, 1952; Shafique, et al., 2016) ,estimation of heritability (Burton and De Vane, 1953), genetic advance, correlation coefficient analysis (Al-Jibouri et al., 1958) and path analysis (Dewey and Lu, 1959).

Correlation coefficient estimates degree of association of different component characters of yield among themselves and with the yield (Hasan and Deb, 2017). The correlation studies between various yields attribute with yield provides a basis for further breeding program (Kishor et al., 2018). Path coefficient analysis measures the direct effect of variable upon another and permits the separation of the correlation coefficient into components of direct and indirect effects (Paneliya et al., 2017; Tiwari et al., 2016; Shengel et al., 2018). Information on the variability and correlation studies among the economic characters of the crop 
is of great value to plant breeders (Swetha et al., 2019; Shedge et al., 2019). It will not only, help to understand the desirable and undesirable relationship of economic characters but also help in assessing the scope of simultaneous improvement of two or more attributes (Shanmugam and kalaimagal, 2019; Sial et al.,2003).

\section{Results and Discussion Analysis of variance}

The mean data for twenty five characters were subjected to analysis of variance for the design of experiment showed that the mean sum of squares due to genotypes were found significant for all the characters under study (Table 1) which indicates that considerable amount of variability is present among the genotypes. Hence there is ample scope for inclusion of promising genotypes in breeding program for yield and its components characters.

\section{Genetic Variability:}

A perusal of variability parameters reveled that wide range of genotypic variance was observed highest genetic variance was recorded for number of seeds per plant (525.27) followed by days to maturity (165.52), days to 50\% flowering (149.62), biological yield (89.59), plant height (40.09), grain yield per plant (21.00), harvest index (20.59), hundred seeds weight (20.43) and chlorophyll index (21.53), (Table 2). whereas low estimates of genetic variability were observed for leaf area index at $55^{\text {th }}$ day (0.04), number of primary branches (0.07), number of seeds per pod (0.07), canopy temperature at vegetative stage $(0.30)$, canopy temperature at pod filling stage (0.59), leaf area index at $108^{\text {th }}$ day, crude fiber $(0.72)$, number of secondary branches (1.24), and phytic acid (1.46). Then moderate estimates of genetic variability were observed for trypsine inhibitor (13.76), Relative water content at $45^{\text {th }}$ day (12.80), specific leaf weight at $110^{\text {th }}$ day (8.81), Relative water content at $105^{\text {th }}$ day (8.04), starch content (5.11), protein content (3.94) and specific leaf weight at $60^{\text {th }}$ day (3.89). This indicates the influence of environment for the expression of most of the characters in present investigation.

Phenotypic variance was also high for number of seeds per plant (705.98) fallowed by days to maturity (166.10), days to $50 \%$ flowering (150.24), biological yield (101.75), plant height (43.54), harvest index (30.47), grain yield per plant (27.02), chlorophyll content (22.64) and hundred seeds weight (20.87). whereas low estimates of phenotypic variance were observed for number of primary branches $(0.10)$, number of seeds per pod (0.10), canopy temperature at vegetative stage (0.61), canopy temperature at pod filling stage (1.01), leaf area index at $55^{\text {th }}$ day $(0.05)$, leaf area index at $108^{\text {th }}$ day (0.6), crude fiber (0.7), number of secondary branches (1.63), and phyticacid (1.54). moderate estimates of phenotypic variance were observed for trypsine inhibitor (13.80), Relative water content at $45^{\text {th }}$ day (16.51), specific leaf weight at $110^{\text {th }}$ day(11.27), Relative water content at $105^{\text {th }}$ day (9.98), starch content (5.40), protein content (4.25), and specific leaf weight at $60^{\text {th }}$ day (5.59), (Table 2).

A wide range of phenotypic coefficient of variation (PCV) was observed for all the traits ranged from canopy temperature at vegetative stage (3.46) to biological yield (25.35). Higher magnitude of PCV were recorded for biological yield (25.35), hundred seeds weight (25.05), grain yield per plant (24.85), number of seeds per plant (22.35), number of seeds per pod (20.28), number of secondary branches (19.49), leaf area index at $55^{\text {th }}$ day (19.23), crude fiber (19.01), leaf area index at $108^{\text {th }}$ day (18.00), low magnitude of PCV were recorded for canopy temperature at pod filling and vegetative stage (2.67) $(2.40)$, relative water content at $45^{\text {th }}$ and $105^{\text {th }}$ day (4.67), (3.97), specific leaf weight at $60^{\text {th }}$ day (4.79) suggested for a limited scope of selection for improvement of these trait, (Table 2). A wide range of genotypic coefficient of variation (GCV) was observed for all the traits ranged from canopy temperature at vegetative stage (2.40) to hundred seeds weight (24.78). Higher magnitude of GCV were recorded for hundred seeds weight (24.78), biological yield (23.79), grain yield per plant (21.92), moderate for number of seeds per plant (19.27), number of seeds per pod (17.60) crude fiber (18.60), number of secondary branches (17.0) and leaf area index at $55^{\text {th }}$ day (16.92), low magnitude of GCV were recorded for canopy temperature at pod filling and vegetative stage (3.48) (3.46), relative water content at $45^{\text {th }}$ and $105^{\text {th }}$ day (5.31) (4.42), specific leaf weight at $60^{\text {th }}$ day (5.74) (Table 2). 
Table 1: Analysis of variance for different characters in chickpea

\begin{tabular}{|l|l|c|c|c|}
\hline \multirow{2}{*}{ SN } & \multirow{2}{*}{ Characters/ traits. } & \multicolumn{3}{|c|}{ Mean Suma of Squares. } \\
\cline { 3 - 5 } & & $\begin{array}{c}\text { Replication } \\
(\mathbf{d f}=\mathbf{0 2})\end{array}$ & $\begin{array}{c}\text { Treatments } \\
\mathbf{( d f}=\mathbf{2 4})\end{array}$ & $\begin{array}{c}\text { Error } \\
\mathbf{d f}=\mathbf{4 8})\end{array}$ \\
\hline 01 & Days to 50\% Flowering & 7.85 & $450.72^{* *}$ & 1.87 \\
\hline 02 & Days to Maturity & 16.69 & $498.30^{*}$ & 1.74 \\
\hline 03 & Plant height & 0.14 & $0.29^{* *}$ & 0.10 \\
\hline 04 & Number of primary branches/plant & 0.26 & $4.90^{* *}$ & 1.17 \\
\hline 05 & Number of secondary branches/plant & 4.72 & $130.62^{* *}$ & 10.34 \\
\hline 06 & Number of seeds/plant & 49.21 & $2117.95^{* *}$ & 542.13 \\
\hline 07 & Number of pods per plant & 0.00 & $0.29^{* *}$ & 0.07 \\
\hline 08 & Hundred seed weight & 1.08 & $62.61^{* *}$ & 1.32 \\
\hline 09 & Grain yield per plant & 43.46 & $81.05^{* *}$ & 18.03 \\
\hline 10 & Biological yield per plant & 74.19 & $305.25^{* *}$ & 36.48 \\
\hline 11 & Harvest index & 9.56 & $91.42^{* *}$ & 29.64 \\
\hline 12 & Chlorophyll index & 4.24 & $67.92^{* *}$ & 3.33 \\
\hline 13 & CT @ Vegetative stage & 0.14 & $1.84^{* *}$ & 0.96 \\
\hline 14 & CT @Pod filling stage & 0.23 & $3.03^{* *}$ & 1.24 \\
\hline 15 & Leaf Area Index @ $55^{\text {th }}$ day & 0.04 & $0.14^{* *}$ & 0.03 \\
\hline 16 & Leaf Area Index $@ 108^{\text {th }}$ day & 0.01 & $0.48^{* *}$ & 0.07 \\
\hline 17 & Specific Leaf Weight $@ 60^{\text {th }}$ day & 6.42 & $16.76^{* *}$ & 5.08 \\
\hline 18 & Specific Leaf Weight $@ 110^{\text {th }}$ day & 6.14 & $33.80^{* *}$ & 7.36 \\
\hline 19 & Relative water Content $@ 45^{\text {th }}$ day & 4.16 & $49.54^{* *}$ & 11.14 \\
\hline 20 & Relative Water Content $@ 105^{\text {th }}$ day & 21.28 & $29.95^{* *}$ & 5.83 \\
\hline 21 & Protein content & 8.50 & $1.47^{* *}$ & 0.62 \\
\hline 22 & Starch content & 10.79 & $0.05^{* *}$ & 0.56 \\
\hline 23 & Crude fiber & 1.51 & $0.21^{* *}$ & 0.06 \\
\hline 24 & Trypsin inhibitor & 27.60 & $6.73^{* *}$ & 0.09 \\
\hline 25 & Phytic acid & 3.07 & $3.35^{* *}$ & 0.16 \\
\hline
\end{tabular}

$* \& * *$ Significant at $\mathrm{P}<0.05$ and $\mathrm{P}<0.01$, respectively

Heritability estimates ranged from 48.2 to 99.7 , heritability was high for Days to maturity (99.7), trypsine inhibitor (99.7), followed by Days to $50 \%$ flowering (99.6), Hundred seed weight (97.9), crude fiber (95.8), chlorophyll index (95.1), phytic acid (94.9), starch content (94.8), protein content (92.7), plant height (92.1), biological yield (88.1), leaf area index at $108^{\text {th }}$ day (85.0), Relative water content (80.5), specific leaf weight at $110^{\text {th }}$ day (78.2), grain yield per plant (77.8), Relative water content at $45^{\text {th }}$ day (77.5), leaf area index at $55^{\text {th }}$ day (77.4), number of secondary branches (76.2), number of seeds per pod (75.3), number of seeds per plant (74.4), specific leaf weight at $60^{\text {th }}$ day (69.7), Harvest index (67.6), number of primary branches (67.1), canopy temperature at both vegetative \& pod filling stage (58.9 \&48.2), Higher values for heritability indicates that it may be due to higher contribution of genotypic components, (Table 2). The Characters with high heritability approximate manifest that contrast in these characters mainly control by heritable constituent, considering that both genetics and environment take part analogous bit part in the pronouncement of characters with quite soaring heritability stipulate that the pronouncement of the character was largely affect by environment more willingly than genetic. character with lofty heritability approximate in broad sense can be employ for genetic refinement as they are least form by the environmental sequel and thus possess a possible for huge genetic resolution (Figure 1). Genetic advance varied from 0.3 (leaf area index at $55^{\text {th }}$ day) to 26.5 ( days to maturity). The maximum genetic advance for days to maturity (26.5) followed by days to $50 \%$. 
Table 2: Estimation of genetic parameters for twenty five characters in twenty five genotypes

\begin{tabular}{|c|c|c|c|c|c|c|c|c|}
\hline SN & Traite & $\begin{array}{l}\text { Genotypic } \\
\text { variance } \sigma^{2} \mathrm{~g}\end{array}$ & $\begin{array}{l}\text { Phenotypi } \\
\text { c variance } \\
\sigma^{2} \mathbf{p}\end{array}$ & GCV & PCV & $\begin{array}{l}\text { Heritab } \\
\text { ility } \\
\mathrm{h}^{2}\end{array}$ & $\begin{array}{l}\text { Genetic } \\
\text { advance } \\
\text { (GA) }\end{array}$ & $\begin{array}{l}\text { GA as } \\
\% \text { of } \\
\text { mean }\end{array}$ \\
\hline 1 & Days to $50 \%$ flowering & 149.62 & 150.24 & 15.32 & 15.35 & 99.6 & 25.1 & 31.5 \\
\hline 2 & Days to maturity & 165.52 & 166.10 & 10.54 & 10.56 & 99.7 & 26.5 & 21.7 \\
\hline 3 & $\begin{array}{lll}\begin{array}{l}\text { Number } \\
\text { branches }\end{array} & \text { of primary } \\
\end{array}$ & 0.07 & 0.10 & 9.90 & 12.90 & 67.1 & 0.4 & 16.7 \\
\hline 4 & $\begin{array}{l}\text { Number of secondary } \\
\text { branches }\end{array}$ & 1.24 & 1.63 & 17.0 & 19.49 & 76.2 & 2.0 & 30.6 \\
\hline 5 & Plant height & 40.09 & 43.54 & 13.98 & 14.57 & 92.1 & 12.5 & 27.6 \\
\hline 6 & Number of seed per plant & 525.27 & 705.98 & 19.27 & 22.35 & 74.4 & 40.7 & 34.2 \\
\hline 7 & Number of seeds per pod & 0.07 & 0.10 & 17.60 & 20.28 & 75.3 & 0.5 & 31.5 \\
\hline 8 & Hundred seed weight & 20.43 & 20.87 & 24.78 & 25.05 & 97.9 & 9.2 & 50.5 \\
\hline 9 & Grain yield per plant & 21.00 & 27.02 & 21.92 & 24.85 & 77.8 & 8.3 & 39.8 \\
\hline 10 & Biological yield & 89.59 & 101.75 & 23.79 & 25.35 & 88.1 & 18.3 & 46.0 \\
\hline 11 & Harvest index & 20.59 & 30.47 & 8.61 & 10.48 & 67.6 & 7.7 & 14.6 \\
\hline 12 & Chlorophyll index & 21.53 & 22.64 & 11.01 & 11.29 & 95.1 & 9.3 & 22.1 \\
\hline 13 & $\begin{array}{l}\text { Canopy temperature - } \\
\text { vegetative stage }\end{array}$ & 0.30 & 0.61 & 2.40 & 3.46 & 48.2 & 0.8 & 3.4 \\
\hline 14 & $\begin{array}{l}\text { Canopy temperature - } \\
\text { pod filling stage }\end{array}$ & 0.59 & 1.01 & 2.67 & 3.48 & 58.9 & 1.2 & 4.2 \\
\hline 15 & $\begin{array}{l}\text { Leaf area index at } 55^{\text {th }} \\
\text { day }\end{array}$ & 0.04 & 0.05 & 16.92 & 19.23 & 77.4 & 0.3 & 30.7 \\
\hline 16 & $\begin{array}{l}\text { Leaf area index } \text { at }{ }^{\text {th }} \\
\text { day }\end{array}$ & 0.13 & 0.6 & 16.59 & 18.00 & 85.00 & 0.7 & 31.5 \\
\hline 17 & $\begin{array}{l}\text { Specific leaf weight at } \\
60^{\text {th }} \text { day }\end{array}$ & 3.89 & 5.59 & 4.79 & 5.74 & 69.7 & 3.4 & 8.2 \\
\hline 18 & $\begin{array}{l}\text { Specific leaf weight at } \\
110^{\text {th }} \text { day }\end{array}$ & 8.81 & 11.27 & 7.17 & 8.10 & 78.2 & 5.4 & 13.1 \\
\hline 19 & 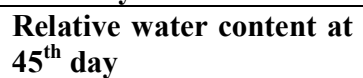 & 12.8 & 16.51 & 4.67 & 5.31 & 77.5 & 6.5 & 8.5 \\
\hline 20 & $\begin{array}{l}\text { Relative water content at } \\
105^{\text {th }} \text { day }\end{array}$ & 8.04 & 9.98 & 3.97 & 4.42 & 80.5 & 5.2 & 7.3 \\
\hline 21 & Protein content & 3.94 & 4.25 & 9.36 & 9.72 & 92.7 & 3.9 & 18.6 \\
\hline 22 & Starch content & 5.11 & 5.40 & 6.14 & 6.31 & 94.8 & 4.5 & 12.3 \\
\hline 23 & Crude fiber & 0.72 & 0.76 & 18.6 & 19.01 & 95.8 & 1.7 & 37.5 \\
\hline 24 & Trypsine inhibitor & 13.76 & 13.80 & 15.6 & 15.62 & 99.7 & 7.6 & 32.1 \\
\hline 25 & Phytic acid & 1.46 & 1.54 & 10.78 & 11.06 & 94.9 & 2.4 & 21.6 \\
\hline
\end{tabular}

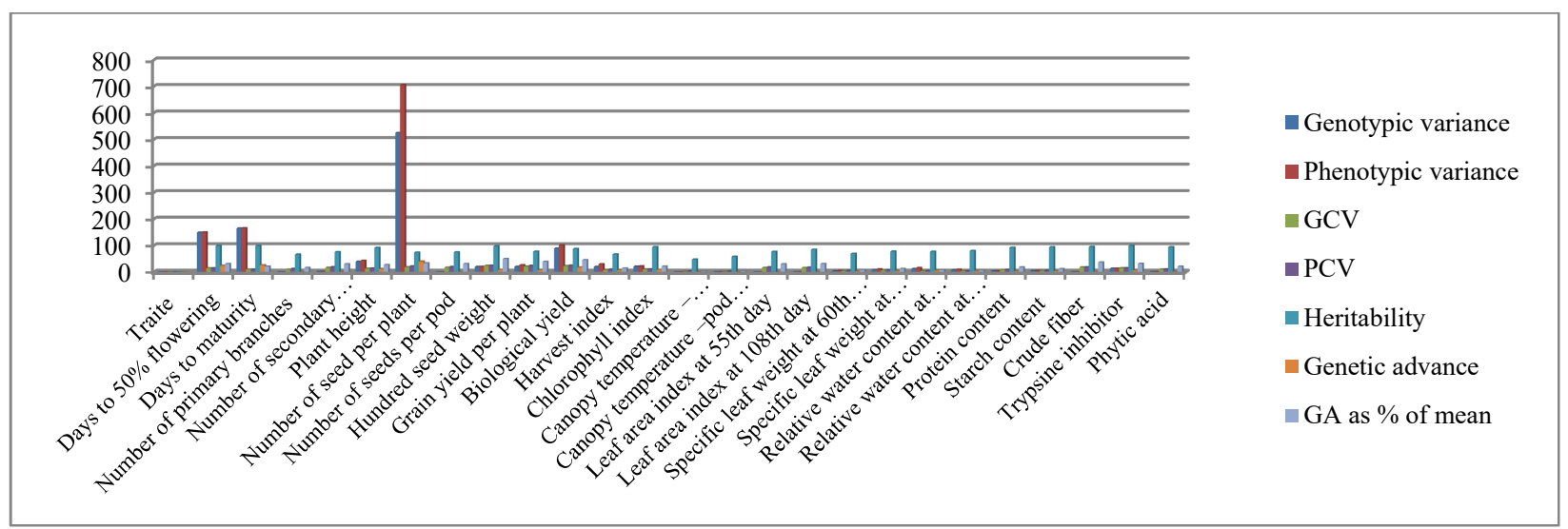

Figure 1: Histogram depicting estimates of genetic parameters for 25 traits in chickpea

\section{3}

Environment Conservation Journal 
Table 3: Genotypic Correlation among different traits in chickpea evaluated under field \& laboratory conditions

\begin{tabular}{|c|c|c|c|c|c|c|c|c|c|c|c|c|c|c|c|c|c|c|c|c|c|c|c|c|c|}
\hline Traits & DF50 & $D M$ & $N P B$ & $N S B$ & $P H$ & $N S P$ & NSPod & $H S W$ & $B M$ & $H I$ & CHI & $C T D_{-} V S$ & CTD_PS & LAI_55 & LAI_108 & $S L W_{-} 60$ & $S L W_{-} 110$ & $R W C_{-} 45$ & $R W C_{-} 105$ & Protein & Starch & Fibre & $T I$ & $P A$ & GYPP \\
\hline DF50 & 1 & & & & & & & & & & & & & & & & & & & & & & & & \\
\hline DM & $0.98^{* *}$ & 1 & & & & & & & & & & & & & & & & & & & & & & & \\
\hline NPB & 0.30 & 0.34 & 1 & & & & & & & & & & & & & & & & & & & & & & \\
\hline NSB & -0.05 & 0.04 & $0.66^{* * *}$ & 1 & & & & & & & & & & & & & & & & & & & & & \\
\hline PH & $0.71^{* *}$ & $0.63^{\text {**t }}$ & 0.32 & -0.21 & 1 & & & & & & & & & & & & & & & & & & & & \\
\hline NSP & 0.36 & 0.38 & -0.05 & 0.20 & -0.06 & 1 & & & & & & & & & & & & & & & & & & & \\
\hline NSPod & 0.19 & 0.14 & -0.04 & -0.14 & 0.12 & 0.19 & 1 & & & & & & & & & & & & & & & & & & \\
\hline HSW & 0.11 & 0.08 & 0.19 & -0.15 & 0.28 & -0.14 & $-0.40^{*}$ & 1 & & & & & & & & & & & & & & & & & \\
\hline BM & 0.37 & $0.40^{*}$ & -0.20 & 0.26 & 0.20 & $0.57^{* * *}$ & -0.22 & 0.02 & 1 & & & & & & & & & & & & & & & & \\
\hline HI & $0.68^{* *}$ & $0.67^{\text {*** }}$ & $0.62^{* * *}$ & -0.07 & $0.55^{* *}$ & 0.14 & 0.27 & 0.11 & -0.22 & 1 & & & & & & & & & & & & & & & \\
\hline CHI & $0.74^{* * *}$ & $0.75^{* * t}$ & 0.24 & 0.06 & $0.43^{*}$ & $0.50^{*}$ & 0.31 & -0.30 & $0.42^{*}$ & $0.57^{* *}$ & 1 & & & & & & & & & & & & & & \\
\hline CTD_VS & -0.11 & -0.06 & 0.20 & 0.32 & 0.00 & -0.28 & 0.13 & 0.20 & $-0.48^{*}$ & 0.35 & $-0.47^{*}$ & 1 & & & & & & & & & & & & & \\
\hline CTD_PS & 0.09 & 0.09 & 0.29 & 0.27 & 0.24 & $0.49^{*}$ & -0.18 & 0.00 & 0.23 & 0.31 & 0.22 & -0.19 & 1 & & & & & & & & & & & & \\
\hline LAI_55 & 0.08 & 0.06 & -0.11 & $0.40^{*}$ & -0.15 & 0.33 & -0.12 & -0.12 & 0.33 & -0.01 & 0.30 & -0.21 & 0.17 & 1 & & & & & & & & & & & \\
\hline SLW_60 & 0.32 & 0.24 & -0.09 & -0.23 & $0.45^{*}$ & $-0.44^{*}$ & $-0.47^{*}$ & $0.58^{* * *}$ & -0.05 & -0.10 & -0.02 & -0.22 & -0.22 & $0.49^{*}$ & 0.11 & 1 & & & & & & & & & \\
\hline SLW_110 & 0.20 & 0.13 & -0.01 & $-0.40^{*}$ & $0.54^{* *}$ & -0.03 & -0.20 & 0.01 & -0.21 & 0.38 & 0.12 & $-0.49^{*}$ & 0.16 & -0.35 & $-0.62^{\text {tw* }}$ & 0.22 & 1 & & & & & & & & \\
\hline RWC_45 & 0.06 & 0.01 & -0.22 & 0.10 & 0.19 & 0.18 & 0.01 & $0.40^{*}$ & 0.21 & -0.18 & 0.04 & 0.14 & 0.31 & 0.35 & 0.31 & $0.57^{* *}$ & -0.08 & 1 & & & & & & & \\
\hline RWC_105 & 0.24 & 0.24 & 0.02 & 0.09 & 0.02 & -0.10 & $0.46^{*}$ & 0.12 & 0.00 & 0.16 & 0.36 & 0.19 & 0.15 & 0.19 & 0.04 & 0.14 & $-0.48^{\prime \prime}$ & $0.46^{*}$ & 1 & & & & & & \\
\hline Protein & -0.27 & -0.26 & 0.28 & -0.04 & -0.19 & -0.08 & $-0.42^{*}$ & -0.08 & -0.26 & -0.20 & 0.02 & $-0.43^{*}$ & $-0.86^{* * *}$ & -0.16 & -0.17 & -0.33 & -0.20 & $-0.51^{\text {tw* }}$ & $-0.63^{\text {tw* }}$ & 1 & & & & & \\
\hline Starch & $0.61^{* *}$ & $0.61^{\text {t*w }}$ & $0.41^{*}$ & $0.61^{* * *}$ & 0.08 & $0.55^{* * *}$ & $-0.44^{*}$ & 0.01 & $0.47^{*}$ & 0.14 & $0.70^{* * *}$ & -0.03 & 0.14 & 0.26 & 0.10 & 0.12 & -0.04 & 0.17 & 0.27 & -0.20 & 1 & & & & \\
\hline Fibre & -0.23 & -0.29 & -0.18 & 0.00 & -0.15 & -0.01 & -0.03 & -0.34 & -0.38 & -0.21 & 0.18 & -0.25 & 0.29 & 0.15 & 0.02 & 0.09 & 0.30 & 0.22 & 0.05 & 0.28 & -0.27 & 1 & & & \\
\hline TI & -0.02 & 0.01 & 0.03 & -0.02 & -0.29 & 0.19 & 0.14 & $-0.44^{*}$ & -0.30 & 0.16 & 0.22 & 0.17 & 0.33 & -0.13 & 0.00 & -0.37 & -0.34 & -0.06 & 0.38 & -0.14 & -0.17 & 0.29 & 1 & & \\
\hline PA & 0.08 & 0.07 & 0.08 & 0.27 & -0.04 & $0.45^{*}$ & 0.14 & -0.06 & 0.18 & 0.12 & 0.32 & -0.29 & 0.31 & -0.03 & -0.16 & -0.15 & 0.16 & 0.26 & 0.32 & 0.12 & 0.15 & 0.33 & -0.18 & 1 & \\
\hline GYPP & $0.60^{* *}$ & $0.62^{* * *}$ & -0.01 & 0.21 & $0.41^{*}$ & $0.67^{* *}$ & -0.11 & 0.06 & $0.93^{* * *}$ & 0.14 & $0.65^{* *}$ & -0.35 & $0.40^{*}$ & 0.37 & 0.21 & -0.07 & -0.04 & 0.18 & 0.04 & -0.29 & $0.55^{* *}$ & $-0.40^{*}$ & -0.21 & 0.28 & 1 \\
\hline
\end{tabular}

$\& * *$ Significant at $\mathrm{P}<0.05$ and $\mathrm{P}<0.01$, respectively

.DF50: Days to 50\% flowering, DM: Days to maturity, PH: Plant height, NPB: No. of primary branch, NSB: No. of secondary branch, NSP: No. of seeds per plant, NSPod: No. of seeds per pod, HSW: 100 seed weight, GYPP: Grain yield per plant, BM: Biomass, HI: Harvest index, CHI: Chlorophyll index, CTD_VS: Canopy temperature depression at vegetative stage, CTD_PS: Canopy temperature depression at pod filling stage, LAI_55: Leaf area index at 55 days, LAI_108: Leaf area index at 108 days, SLW_60: Specific leaf weight at 60 days, SLW_110: Specific leaf weight at 110 days, RWC_45: Relative water content at 45 days, RWC_105: Relative water content at 105 days, TI: Trypsine Inhibitor, PA: Phytic acid content 
Table 4: Phenotypic Correlation among different traits in chickpea evaluated under field \& laboratory conditions

\begin{tabular}{|c|c|c|c|c|c|c|c|c|c|c|c|c|c|c|c|c|c|c|c|c|c|c|c|c|c|}
\hline Traits & DF50 & $D M$ & $N P B$ & $N S B$ & $P H$ & $N S P$ & NSPod & $H S W$ & $B M$ & $H I$ & CHI & CTD_VS & $C T D_{-} P S$ & LAI_55 & LAI_ 108 & SLW_60 & $S L W_{-} I 10$ & $R W C_{-} 45$ & $R W C_{-} 105$ & Protein & Starch & Fibre & $T I$ & $P A$ & GYPP \\
\hline DF50 & 1 & & & & & & & & & & & & & & & & & & & & & & & & \\
\hline DM & $0.98^{* *}$ & 1 & & & & & & & & & & & & & & & & & & & & & & & \\
\hline NPB & 0.26 & 0.29 & 1 & & & & & & & & & & & & & & & & & & & & & & \\
\hline NSB & -0.05 & 0.04 & $0.57^{* *}$ & 1 & & & & & & & & & & & & & & & & & & & & & \\
\hline PH & $0.67^{* * *}$ & $0.60^{* *}$ & 0.24 & -0.20 & 1 & & & & & & & & & & & & & & & & & & & & \\
\hline NSP & 0.32 & 0.33 & 0.02 & 0.19 & -0.05 & 1 & & & & & & & & & & & & & & & & & & & \\
\hline NSPod & 0.12 & 0.10 & -0.04 & -0.11 & 0.09 & 0.13 & 1 & & & & & & & & & & & & & & & & & & \\
\hline HSW & 0.13 & 0.08 & 0.16 & -0.12 & 0.28 & -0.13 & -0.31 & 1 & & & & & & & & & & & & & & & & & \\
\hline BM & 0.36 & 0.39 & -0.06 & 0.28 & 0.19 & $0.55^{* *}$ & -0.16 & 0.02 & 1 & & & & & & & & & & & & & & & & \\
\hline HI & $0.54^{* * *}$ & $0.56^{* *}$ & $0.44^{*}$ & 0.00 & $0.44^{*}$ & 0.18 & 0.12 & 0.12 & -0.15 & 1 & & & & & & & & & & & & & & & \\
\hline CHI & $0.72^{* * *}$ & $0.73^{* * *}$ & 0.21 & 0.04 & $0.40^{*}$ & $0.43^{*}$ & 0.19 & -0.27 & $0.41^{*}$ & $0.46^{*}$ & 1 & & & & & & & & & & & & & & \\
\hline CTD_VS & -0.08 & -0.04 & 0.20 & 0.18 & -0.03 & -0.13 & 0.12 & 0.14 & -0.24 & 0.10 & -0.32 & 1 & & & & & & & & & & & & & \\
\hline CTD_PS & 0.10 & 0.09 & 0.16 & 0.25 & 0.19 & $0.41^{*}$ & $\begin{array}{l}-0.07 \\
\end{array}$ & -0.02 & 0.20 & 0.26 & 0.22 & 0.00 & 1 & & & & & & & & & & & & \\
\hline LAI_55 & 0.02 & 0.03 & -0.15 & 0.27 & -0.18 & 0.27 & -0.22 & -0.08 & 0.30 & -0.05 & 0.19 & -0.18 & 0.15 & 1 & & & & & & & & & & & \\
\hline LAI_108 & -0.05 & -0.01 & -0.01 & 0.27 & -0.19 & 0.17 & 0.02 & 0.10 & 0.24 & -0.12 & -0.13 & $\begin{array}{l}-0.11 \\
\end{array}$ & $\begin{array}{l}-0.01 \\
\end{array}$ & $0.51^{* *}$ & 1 & & & & & & & & & & \\
\hline SLW_60 & 0.30 & 0.22 & 0.02 & -0.11 & 0.39 & -0.25 & -0.32 & $0.45^{*}$ & -0.01 & -0.02 & 0.03 & -0.05 & -0.10 & $0.43^{*}$ & 0.17 & 1 & & & & & & & & & \\
\hline SLW_110 & 0.19 & 0.12 & -0.04 & -0.32 & $0.47^{*}$ & 0.01 & -0.12 & -0.02 & -0.18 & 0.30 & 0.13 & -0.26 & 0.13 & -0.20 & $-0.45^{*}$ & 0.18 & 1 & & & & & & & & \\
\hline RWC_45 & 0.05 & 0.01 & -0.20 & 0.10 & 0.15 & 0.13 & 0.00 & 0.35 & 0.14 & -0.10 & 0.02 & 0.08 & 0.28 & 0.33 & 0.23 & $0.40^{*}$ & -0.07 & 1 & & & & & & & \\
\hline RWC_105 & 0.20 & 0.22 & 0.04 & 0.11 & -0.01 & -0.07 & 0.32 & 0.12 & 0.02 & 0.15 & 0.31 & 0.08 & 0.15 & 0.12 & 0.00 & 0.17 & -0.32 & 0.36 & 1 & & & & & & \\
\hline Protein & -0.27 & -0.24 & -0.08 & -0.16 & -0.18 & -0.10 & -0.25 & -0.12 & -0.21 & 0.10 & -0.08 & -0.15 & -0.39 & 0.10 & -0.23 & -0.17 & 0.12 & -0.35 & $-0.45^{*}$ & 1 & & & & & \\
\hline Starch & $0.52^{* *}$ & $0.59^{* * *}$ & 0.30 & 0.38 & 0.08 & 0.31 & -0.34 & 0.03 & $0.49^{* * *}$ & 0.15 & $0.49^{* * *}$ & 0.03 & 0.07 & 0.26 & -0.06 & 0.19 & 0.06 & 0.01 & 0.10 & -0.18 & 1 & & & & \\
\hline Fibre & -0.26 & -0.30 & -0.29 & -0.15 & -0.06 & -0.16 & 0.05 & -0.32 & -0.35 & 0.07 & 0.11 & -0.26 & 0.27 & 0.38 & -0.14 & 0.07 & 0.27 & 0.20 & 0.08 & 0.27 & -0.27 & 1 & & & \\
\hline TI & -0.03 & 0.00 & 0.01 & -0.09 & -0.18 & -0.10 & 0.34 & $-0.42^{*}$ & -0.27 & 0.20 & 0.20 & 0.20 & 0.26 & -0.01 & -0.08 & -0.38 & -0.36 & -0.15 & 0.29 & -0.14 & -0.15 & 0.29 & 1 & & \\
\hline PA & 0.08 & 0.06 & -0.05 & 0.12 & 0.02 & 0.28 & 0.19 & -0.11 & 0.17 & 0.20 & 0.29 & -0.22 & 0.17 & 0.23 & -0.18 & -0.09 & 0.31 & 0.16 & 0.20 & 0.10 & 0.18 & 0.33 & -0.20 & 1 & \\
\hline GYPP & $0.53^{* *}$ & $0.56^{* *}$ & 0.11 & 0.26 & 0.36 & $0.63^{* * *}$ & -0.11 & 0.06 & $0.91^{* *}$ & 0.26 & $0.59^{* * *}$ & -0.18 & 0.34 & 0.29 & 0.19 & -0.01 & -0.03 & 0.12 & 0.06 & -0.18 & $0.51^{\prime * *}$ & -0.30 & -0.18 & 0.24 & 1 \\
\hline
\end{tabular}

$* \& * *$ Significant at $\mathbf{P}<0.05$ and $\mathbf{P}<0.01$, respectively

DF50: Days to 50\% flowering, DM: Days to maturity, PH: Plant height, NPB: No. of primary branch, NSB: No. of secondary branch, NSP: No. of seeds per plant, NSPod: No. of seeds per pod, HSW: 100 seed weight, GYPP: Grain yield per plant, BM: Biomass, HI: Harvest index, CHI: Chlorophyll index, CTD_VS: Canopy temperature depression at vegetative stage, CTD PS: Canopy temperature depression at pod filling stage, LAI 55: Leaf area index at 55 days, LAI 108: Leaf area index at 108 days, SLW 60: Specific leaf weight at 60 days, SLW 110: Specific leaf weight at 110 days, RWC_45: Relative water content at 45 days, RWC_105: Relative water content at 105 days, TI: Trypsine Inhibitor, PA: Phytic acid content 


\section{Table 5: Direct (in bold) and indirect effects of 23 traits on grain yield in chickpea evaluated}

\begin{tabular}{|c|c|c|c|c|c|c|c|c|c|c|c|c|c|c|c|c|c|c|c|c|c|c|c|c|}
\hline & DF50 & PH & NPB & NSB & NSP & NSPod & HSW & HI & CHI & CTD_VS & CTD_PS & LAI_55 & LAI_108 & SLW_60 & SLW_110 & RWC_45 & RWC_105 & Protein & Starch & Fibre & $\mathrm{TI}$ & PA & GYPP & Residual \\
\hline DF50 & -0.815 & 0.633 & -0.171 & -0.023 & 0.184 & -0.067 & 0.034 & 0.113 & 0.523 & 0.003 & -0.020 & 0.000 & 0.000 & 0.012 & -0.027 & -0.018 & -0.010 & 0.063 & 0.022 & 0.095 & -0.016 & 0.013 & 0.530 & 0.048 \\
\hline PH & -0.554 & 0.932 & -0.157 & -0.107 & -0.023 & -0.050 & 0.079 & 0.093 & 0.294 & 0.001 & -0.041 & 0.000 & -0.017 & 0.017 & -0.067 & -0.046 & 0.000 & 0.040 & 0.004 & 0.023 & -0.059 & 0.000 & 0.360 & 0.048 \\
\hline NPB & -0.204 & 0.214 & -0.682 & 0.321 & 0.012 & 0.017 & 0.049 & 0.088 & 0.143 & -0.007 & -0.039 & 0.000 & -0.001 & 0.001 & 0.006 & 0.061 & -0.002 & 0.018 & 0.012 & 0.111 & 0.003 & -0.011 & 0.110 & 0.048 \\
\hline NSB & 0.033 & -0.177 & -0.389 & 0.563 & 0.115 & 0.033 & -0.037 & 0.002 & 0.036 & -0.007 & -0.059 & -0.001 & 0.034 & -0.005 & 0.049 & -0.031 & -0.005 & 0.036 & 0.016 & 0.057 & -0.031 & 0.026 & 0.260 & 0.048 \\
\hline NSP & -0.261 & -0.037 & -0.014 & 0.113 & 0.576 & -0.059 & -0.043 & 0.037 & 0.308 & 0.004 & -0.098 & -0.001 & 0.022 & -0.011 & 0.000 & -0.043 & 0.003 & 0.022 & 0.013 & 0.061 & -0.034 & 0.071 & 0.630 & 0.048 \\
\hline NSPod & -0.130 & 0.112 & 0.027 & -0.045 & 0.081 & -0.419 & -0.101 & 0.033 & 0.179 & -0.005 & 0.027 & 0.000 & 0.016 & -0.016 & 0.021 & -0.003 & -0.015 & 0.058 & -0.010 & -0.015 & 0.087 & 0.029 & -0.090 & 0.048 \\
\hline HSW & -0.090 & 0.242 & -0.109 & -0.068 & -0.081 & 0.138 & 0.305 & 0.021 & -0.208 & -0.005 & 0.000 & 0.000 & 0.006 & 0.021 & 0.001 & -0.107 & -0.004 & 0.025 & 0.000 & 0.118 & -0.124 & -0.024 & 0.060 & 0.048 \\
\hline $\mathrm{HI}$ & -0.448 & 0.419 & -0.293 & 0.006 & 0.104 & -0.067 & 0.031 & 0.206 & 0.337 & -0.004 & -0.059 & 0.000 & -0.009 & -0.002 & -0.043 & 0.028 & -0.007 & -0.020 & 0.007 & -0.027 & 0.059 & 0.045 & 0.260 & 0.048 \\
\hline $\mathrm{CHI}$ & -0.595 & 0.382 & -0.136 & 0.028 & 0.248 & -0.105 & -0.089 & 0.097 & 0.716 & 0.010 & -0.046 & -0.001 & -0.007 & 0.000 & -0.016 & -0.006 & -0.014 & 0.020 & 0.021 & -0.042 & 0.053 & 0.063 & 0.580 & 0.048 \\
\hline CTD_VS & 0.057 & -0.019 & -0.136 & 0.101 & -0.069 & -0.063 & 0.040 & 0.025 & -0.208 & -0.036 & 0.005 & 0.000 & -0.009 & -0.003 & 0.040 & -0.024 & -0.004 & 0.034 & 0.002 & 0.099 & 0.059 & -0.061 & -0.170 & 0.048 \\
\hline CTD_PS & -0.065 & 0.158 & -0.109 & 0.135 & 0.230 & 0.046 & 0.000 & 0.049 & 0.136 & 0.001 & -0.244 & 0.000 & -0.006 & -0.004 & -0.021 & -0.083 & -0.006 & 0.085 & 0.002 & -0.099 & 0.084 & 0.050 & 0.340 & 0.048 \\
\hline LAI_55 & -0.057 & -0.112 & 0.096 & 0.152 & 0.155 & 0.025 & -0.034 & 0.002 & 0.179 & 0.005 & -0.022 & -0.003 & 0.069 & 0.016 & 0.033 & -0.098 & -0.007 & -0.016 & 0.012 & -0.133 & -0.012 & 0.040 & 0.290 & 0.048 \\
\hline LAI_108 & 0.000 & -0.130 & 0.007 & 0.158 & 0.104 & -0.054 & 0.015 & -0.014 & -0.043 & 0.003 & 0.012 & -0.002 & 0.121 & 0.005 & 0.067 & -0.067 & -0.002 & 0.054 & 0.000 & 0.050 & -0.034 & -0.058 & 0.190 & 0.048 \\
\hline SLW_60 & -0.220 & 0.345 & -0.014 & -0.068 & -0.144 & 0.146 & 0.141 & -0.008 & 0.000 & 0.003 & 0.020 & -0.001 & 0.015 & 0.046 & -0.028 & -0.122 & -0.007 & 0.036 & 0.006 & -0.027 & -0.112 & -0.016 & -0.010 & 0.048 \\
\hline SLW_110 & -0.147 & 0.419 & 0.027 & -0.186 & 0.000 & 0.059 & -0.003 & 0.060 & 0.079 & 0.010 & -0.034 & 0.001 & -0.055 & 0.009 & -0.150 & 0.021 & 0.014 & -0.029 & 0.002 & -0.103 & -0.109 & 0.085 & -0.030 & 0.048 \\
\hline RWC_45 & $\begin{array}{l}-0.049 \\
\end{array}$ & 0.140 & 0.136 & 0.056 & 0.081 & -0.004 & 0.107 & -0.019 & 0.014 & -0.003 & $\begin{array}{l}-0.066 \\
\end{array}$ & $\begin{array}{l}-0.001 \\
\end{array}$ & 0.027 & 0.018 & 0.010 & -0.306 & -0.016 & 0.078 & $\begin{array}{ll}0.001 \\
\end{array}$ & -0.076 & -0.050 & 0.040 & 0.120 & 0.048 \\
\hline RWC_105 & -0.179 & 0.009 & -0.027 & 0.068 & -0.035 & -0.146 & 0.031 & 0.035 & 0.236 & -0.004 & $\begin{array}{l}-0.032 \\
\end{array}$ & -0.001 & 0.006 & 0.007 & 0.049 & -0.110 & -0.043 & 0.103 & 0.005 & -0.030 & 0.084 & 0.045 & 0.070 & 0.048 \\
\hline Protein & 0.228 & -0.168 & 0.055 & -0.090 & -0.058 & 0.109 & -0.034 & 0.019 & -0.064 & 0.005 & 0.093 & 0.000 & -0.029 & -0.007 & -0.019 & 0.107 & 0.020 & -0.224 & -0.008 & -0.103 & -0.040 & 0.029 & -0.180 & 0.048 \\
\hline Starch & -0.440 & 0.093 & -0.198 & 0.220 & 0.184 & 0.100 & 0.003 & 0.035 & 0.365 & -0.002 & -0.010 & -0.001 & 0.000 & 0.007 & -0.006 & -0.006 & -0.005 & 0.043 & 0.040 & 0.103 & -0.053 & 0.037 & 0.510 & 0.048 \\
\hline Fibre & 0.204 & -0.056 & 0.198 & -0.084 & -0.092 & -0.017 & -0.095 & 0.014 & 0.079 & 0.009 & -0.063 & -0.001 & -0.016 & 0.003 & -0.040 & -0.061 & -0.003 & -0.060 & -0.011 & -0.381 & 0.090 & 0.085 & -0.300 & 0.048 \\
\hline $\mathrm{TI}$ & 0.041 & -0.177 & -0.007 & -0.056 & -0.063 & -0.117 & -0.122 & 0.039 & 0.122 & -0.007 & -0.066 & 0.000 & -0.013 & -0.016 & 0.052 & 0.049 & -0.012 & 0.029 & -0.007 & -0.111 & 0.310 & -0.048 & -0.180 & 0.048 \\
\hline PA & -0.041 & 0.000 & 0.027 & 0.056 & 0.155 & -0.046 & -0.027 & 0.035 & 0.172 & 0.008 & -0.046 & 0.000 & -0.027 & -0.003 & -0.048 & -0.046 & -0.007 & -0.025 & 0.006 & -0.122 & 0.056 & 0.264 & .230 & 104 \\
\hline
\end{tabular}

Residual effect: 0.048, DF50: Days to 50\% flowering, PH: Plant height, NPB: No. of primary branch, NSP: No. of seeds per plant, NSPod: No. of seeds per pod, HSW: 100 seed weight, GYPP: Grain yield per plant, HI: Harvest index, CHI: Chlorophyll index, CTD VS: Canopy temperature depression at vegetative stage, CTD PS: Canopy temperature depression at pod filling stage, LAI_55: Leaf area index at 55 days, LAI_108: Leaf area index at 108 days, SLW_60: Specific leaf weight at 60 days, SLW_110: Specific leaf weight at 110 days, RWC_45:

Relative water content at 45 days, RWC_105: Relative water content at 105 days, TI: Trypsine Inhibitor, PA: Phytic acid content 


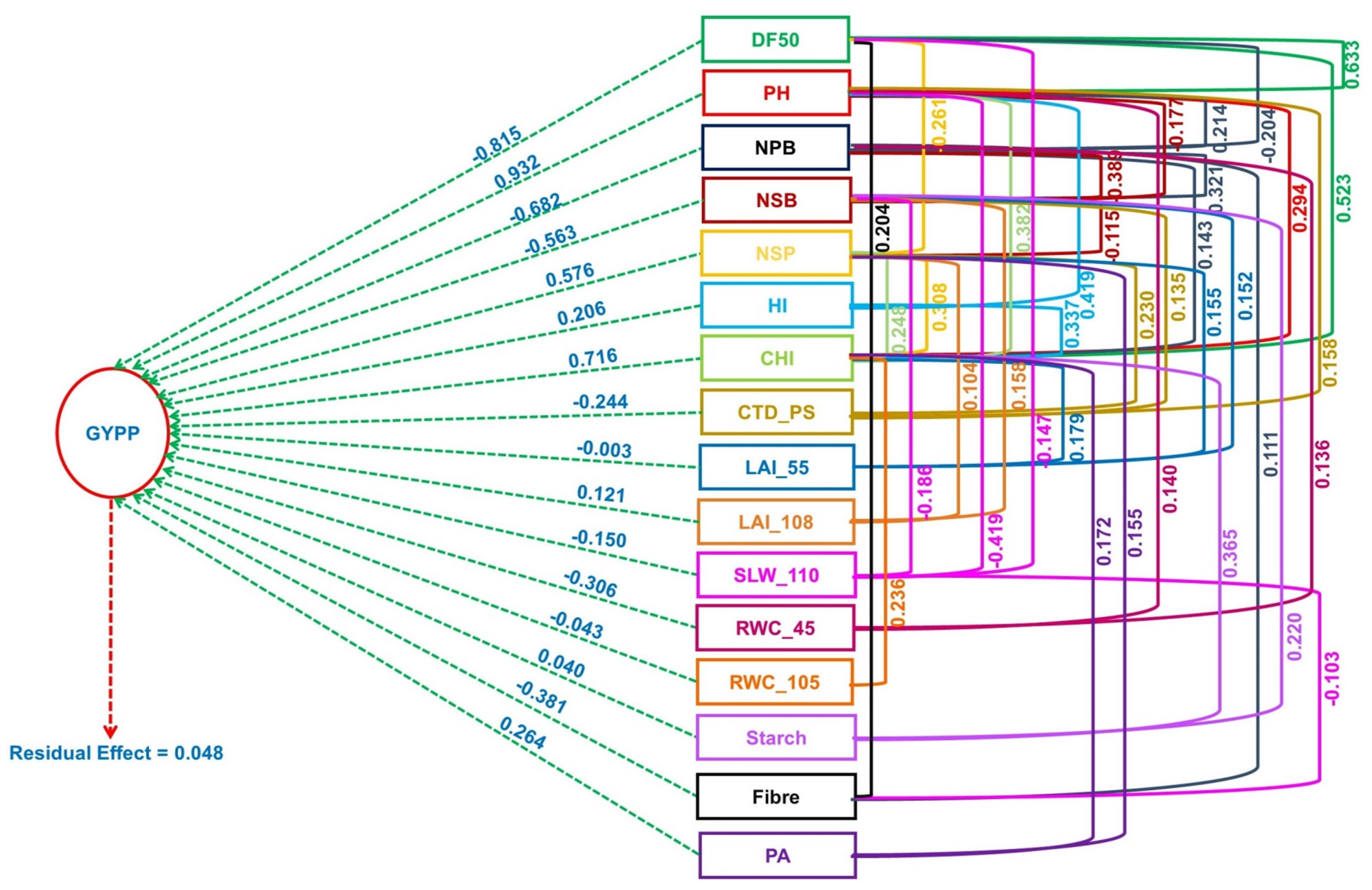

Figure 2: Phenotypic path diagram for seed yield per plant 
Residual effect: 0.048 ,

DF50: Days to 50\% flowering, PH: Plant height, NPB: No. of primary branch, NSP: No. of seeds per plant, GYPP: Grain yield per plant, HI: Harvest index, CHI: Chlorophyll index, , CTD_PS: Canopy temperature depression at pod filling stage, LAI_55: Leaf area index at 55 days, LAI_108: Leaf area index at 108 days, , SLW_110: Specific leaf weight at 110 days, RWC_45: Relative water content at 45 days, RWC_105: Relative water content at 105 days, TI: Trypsine Inhibitor, PA: Phytic acid content, SC: starch content, $\mathrm{CF}$ : crude fiber flowering (25.1), biological yield (18.3), plant height (12.5), (Table 2). Genetic advance as per percent of mean varied from 8.2 (specific leaf weight at $60^{\text {th }}$ day) to $50.5 \%$ (hundred seed weight ) . Genetic advance as per percent of mean was high for Hundred seed weight (50.5), biological yield (46.0), grain yield per plant (39.8), crude fiber (37.5), number of seeds per plant (34.2), and moderate for trypsine inhibitor (32.1), days to $50 \%$ flowering (31.5), number of secondary branches (30.6)plant height (27.6), chlorophyll index (22.1), than lower for Relative water content at $45^{\text {th }}$ day (8.5), specific leaf weight at $60^{\text {th }}$ day (8.2), Relative water content at $105^{\text {th }}$ day (7.3), canopy temperature at vegetative and pod filling stage (4.2 \&3.4) (Table 2).

\section{Correlation coefficient analysis:}

The present study in genotypic correlation grain yield per plant showed high significant and positive association with biological yield $(0.93 * *)$, days to $50 \%$ flowering $(0.60 * *)$, days to maturity $(0.62 * *)$, number of seeds per plant $\left(0.67^{* *}\right)$, chlorophyll index $(0.65 * *)$, starch content $\left(0.55^{* *}\right)$, Plant height $\left(0.41^{*}\right)$, and canopy temperature at pod filling stage $\left(0.40^{*}\right)$. It also showed positive but non-significant association with leaf area index at $55^{\text {th }}$ day (0.37), phytic acid (0.28), number of secondary branches $(0.21)$, leaf area index at $108^{\text {th }}$ day $(0.21)$, relative water content at $45^{\text {th }}$ day $(0.18)$, harvest index $(0.14)$, hundred seed weight $(0.06)$, and relative water content at $105^{\text {th }}$ day (0.04), (Table 3 ). In phenotypic correlation grain yield per plant exhibited positive and significant association with biological yield $\left(0.91^{* *}\right)$, number of seeds per plant $(0.63 * *)$, days to maturity $\left(0.56^{* *}\right)$, days to $50 \%$ flowering $(0.53 * *)$, chlorophyll index $(0.59 * *)$, and starch content $(0.51 * *)$. It also showed positive and non-significant association with number of primary branches $(0.11)$, number of secondary branches $(0.26)$, plant height $(0.36)$, hundred seed weight (0.06), harvest index (0.26), canopy temperature at pod filling stage (0.34), leaf area index at $55^{\text {th }}$ and $108^{\text {th }}$ day $(0.29)(0.19)$, relative water content at $45^{\text {th }}$ and $105^{\text {th }}$ day $(0.12)$ (0.06), and phytic acid (0.24) (Table 4).

\section{Path coefficient analysis}

The highest direct and positive effect on seed yield was exhibited by Hundred seed weight (0.305), Harvest index (0.206), Chlorophyll content (0.716), Leaf area index at $108^{\text {th }}$ day $(0.121)$, Specific leaf weight at $60^{\text {th }}$ day $(0.046)$, Starch content $(0.040)$, Trypsin inhibitor (0.310), Phytic acid (0.264), Number of seeds per plant (0.576), Number of secondary branches (0.563), Plant height (0.932), The negative direct effect on seed yield was exhibited by Days to $50 \%$ flowering $(-0.815)$, Number of primary branches (-0.682), Number of seeds per pod (-0.419), Canopy temperature at vegetative and pod filling stage $(-0.036)(-0.244)$, Leaf area index at $55^{\text {th }}$ day $\quad(-0.003)$, Specific leaf weight at $110^{\text {th }}$ day $(-0.150)$, Relative water content at $45^{\text {th }}$ day and $105^{\text {th }}$ day $(-0.306)(-0.043)$, Protein content $(-0.224)$, Crude fiber $(-0.381)$. Thus these characters turned out to be the major component of seed yield (Table 5 \& Figure 1).

\section{Conclusion}

It is concluded from the present study that all the 25 genotypes of chickpea showed significant differences. Genotypes C-18106, C-18122, C18103, and C-18101 showed better performance for seed yield, C-18123, ICC-15896, C-18102 and GPF-02 showed pod borer resistance. Biological yield, Harvest index, Hundred seed weight, Leaf area index at $108^{\text {th }}$ day, Specific leaf weight at $60^{\text {th }}$ day, Starch content, Trypsin inhibitor, Phytic acid number of seeds per plant and chlorophyll index have positive significant correlation and direct effect on seed yield, the genotypes with these characters can be used for further improvement and development of chickpea.

\section{References}

Al-Jibouri, H. A., Miller, P. A., \& Robinson, H. F. (1958). Genotypic and environmental variances and covariances in an upland Cotton cross of interspecific origin 1. Agronomy journal, 50(10), 633-636.

Arora, P. P., \& Jeena, A. S. (2001). Genetic variability studies in chickpea. Legume Research-An International Journal, 24(2), 137-138. 
Awol, M., \& Alise, F. (2018). Correlation and path analysis in yield and yield components in Ethiopian chick pea land races. Journal of food and agriculture 12(6), 35-37.

Banik, M., Deore, G. N., Mandal, A. K., \& Mhase, L. B. (2018). Genetic Variability and Heritability Studies in Chickpea (Cicer arietinum L.). Current Journal of Applied Science and Technology, 31(1), 1-6.

Burton, G. W. (1952). Quantitative inheritance in grasses. Pro VI Int Grassl Cong, 1952, 277-283.

Burton, G. W., \& Devane, D. E. (1953). Estimating heritability in tall fescue (Festuca arundinacea) from replicated clonal material 1. Agronomy journal, 45(10), 478-481.

Chopdar, D. K., Bharti, B., Sharma, P. P., Dubey R. B., Rajendra \&Meena, B. L. (2017) .Studies on genetic variability, character association and path analysis for yield and its contributing traits in chickpea [Cicer arietinum (L.)] Legume Research, (3603) 824-829.

Dehal, I. B., Kalia, R., \& Kumar, B. (2016). Genetic estimates and path coefficient analysis in chickpea (Cicer arietinum L.) under normal and late sown environments. Legume Research-An International Journal, 39(4), 510-516.

Dewey, D. R., \& Lu, K. (1959). A Correlation and PathCoefficient Analysis of Components of Crested Wheatgrass Seed Production 1. Agronomy journal, 51(9), $515-518$

Directorate of economics and statistics, 2019: The annual report on crop production, published by ministry of agriculture and farmers welfare.

Fiaz, S., Aslam, A., Fahad, M. W., Aamir, R. \& Ikram, B. (2016). Interrelationships among yield and yield contributing traits in chickpea (Cicer arietinum L.) International Journal of Biosciences 9(2), 49-57.

Fisher, R. A., \& Yates, F. (1963). Statistical tables for biological, agricultural and medical research, edited by ra fisher and $f$. yates. Edinburgh: Oliver and Boyd.

Gediya, L. N., Patel, D. A., Kumar, S., Kumar, D., Parmar, D. J., \& Patel, S. S. (2019). Phenotypic variability, path analysis and molecular diversity analysis in chickpea (Cicer arietinum L.). Vegetos, 32(2), 167-180.

Hasan, M. T., \& Deb, A. C. (2017). Assessment of genetic variability, heritability, character association and selection indexes in chickpea (Cicer arietinum L.). Int $J$ Biosci, 10(2), 111-129.

Kaur, J., \& Bhardwaj, N. (2019). Correlation studies for yield and its components in chickpea under low input conditions. Himachal Journal of Agricultural Research, 45(1\&2), 15-19.
Kishor, L., Swarup. I., Nehra, A., Kirar, G., \& Jeetarwal, R.C. (2018) Genetic Variability, Heritability and Genetic Advance Analysis in Chickpea (Cicer arietinum L.). International Journal of Pure and Applied Bioscience 6 (4), 141-144.

Kumar, A., Kumar, A., Yadav, A. K., Nath, S., Yadav, J. K., \& Kumar, D. (2018). Correlation and path coefficient analysis for various quantitative traits in chickpea (Cicer arietinum L.). Journal of Pharmacognosy and Phytochemistry, 26952699.

Kumar, S., Suresh, B. G., Kumar, A., \& Lavanya, G. R. (2019). Genetic variability in chickpea (Cicer arietinum L.) under heat stress condition. Current Journal of Applied Science and Technology, 38(6), 1-10.

Maloo, S. R., \& Sharma, P. P. (1987). Estimation of variability parameters and path coefficient analysis in gram (Cicer arietinum L.). Madras Agric. J, 74, 381-386.

Manasa, B., Priya, M.S., Jayalakshmi, J., \& Umamaheswari, P. (2019). Character Association and Path Coefficient Analysis in Extra Large and Large Seeded Kabuli Chickpea (Cicer arietinum L.) International Journal of Pure and Applied Science .7(5), 166-171.

Muhammad, A., Zubair, M., \&Ghafoor, A. (2003). Genetic variability and correlation studies in chickpea (Cicer arietinum L.). Pak. J. Bot, 35(4), 605-611.

Paneliya, M. R., Mehta, D. R., Jalu, R. K., \& Chetariya, C. P. (2017). Correlation and path coefficient analysis in Desi Chickpea (Cicer arietinum L.) International Journal of Pure and Applied Sciences 5(4), 425-432.

Sain, M., Singh, A., Kaur, A., \& Zalpouri, R. (2020). Metaphysical energy based sustainable yogic farming for enhanced productivity and farmers' well being: A Review. Environment Conservation Journal, 21(3), 63-68.

Saroj, S. K., Singh, M. N., Kumar, R. A. V. I. N. D. R. A., Singh, T. E. J. V. E. E. R., \& Singh, M. K. (2013). Genetic variability, correlation and path analysis for yield attributes in pigeonpea. The bioscan, 8(3), 941-944.

Shafique, M. S., Muhammad, A., Zafar, M., Muhammad, A., Awais, S., \& Ahmad, M. I. (2016). Genetic variability and interrelationship of various agronomic traits using correlation and path analysis in chickpea (Cicer arietinum L.). Academia Journal of Agricultural Research, 4(2), 8285 .

Shanmugam, M., \& Kalaimagal T. (2019). Genetic Variability, Correlation and Path Coefficient Analysis in Chickpea (Cicer arietinum L.) for Yield and its Component Traits Int.J.Curr.Microbiol.App.Sci. 8(5):1801-1808.

Shedge, P.J., Patil, D.K., \& Dawane, J.K. (2019). Correlation and Path Coefficient Analysis of Yield and Yield 
Components in Chickpea (Cicer arietinum L.) International Journal International Journal of Current Microbiology and Applied Sciences, 8(5), 82-85.

Sial, P. (2003). Studies on genetic variability, heritability and genetic advance in chickpea (Cicer arietinum L.). Environment and Ecology, 21(1), 210-213.

Singh, A., \& Sandhu, J. S. (2008).Correlation and path analysis in chickpea under different environments. Journal of Food Legume, 21(2), 145-148.

Swetha, P. B., \& Lavanya, G. R. (2019). Genetic variability, heritability and character association for yield and component characters in chickpea (Cicer arietinum
L.). Journal of Pharmacognosy and Phytochemistry, 8(5), 161-163.

Tiwari, A., Babbar, A., \& Pal, N. (2016). Genetic variability, correlation and path analysis in yield and yield components in chickpea (Cicer arietinum L.) genotypes under late sown condition. International Journal of Agriculture Research, 8(54), 2884-2886.

Vavilov, N. I. (1926). Studies on the origins of cultivated plants. Bullet Applied Plant Breeding, 16(1), 245.

Wright, S. (1921) Correlation and causation. Journal Agriculture Research, 20(1), 557-585. 